\title{
Combination of natural killer cell-based immunotherapy and irreversible electroporation for the treatment of hepatocellular carcinoma
}

\author{
Aydin Eresen $^{1,2}$, Jia Yang ${ }^{1}$, Alessandro Scotti ${ }^{3,4}$, Kejia Cai ${ }^{3,4}$, Vahid Yaghmai ${ }^{2,5}$, Zhuoli Zhang ${ }^{1,2,5,6}$ \\ ${ }^{1}$ Department of Radiology, Feinberg School of Medicine, Northwestern University, Chicago, IL, USA; ${ }^{2}$ Department of Radiological Sciences, \\ University of California Irvine, Irvine, CA, USA; ${ }^{3}$ Department of Radiology, University of Illinois at Chicago, Chicago, IL, USA; ${ }^{4}$ Department of \\ Bioengineering, University of Illinois at Chicago, Chicago, IL, USA; ${ }^{5}$ Chao Family Comprehensive Cancer Center, University of California, Irvine, \\ CA, USA; ${ }^{6}$ Robert H. Lurie Comprehensive Cancer Center of Northwestern University, Chicago, IL, USA \\ Contributions: (I) Conception and design: A Eresen, V Yaghmai, Z Zhang; (II) Administrative support: A Eresen, Z Zhang; (III) Provision of study \\ materials or patients: J Yang, V Yaghmai, K Cai; (IV) Collection and assembly of data: A Eresen; (V) Data analysis and interpretation: All authors; \\ (VI) Manuscript writing: All authors; (VII) Final approval of manuscript: All authors. \\ Correspondence to: Zhuoli Zhang, MD, PhD. Department of Radiological Sciences, School of Medicine, University of California Irvine, 839 Health \\ Sciences Rd., Irvine, CA 92617, USA. Email: zhuoliz1@hs.uci.edu.
}

\begin{abstract}
Hepatocellular carcinoma (HCC) is among the most lethal cancer types despite great advancement in overall survival of the patients over the last decades. Surgical resection or partial hepatectomy has been approved as the curative treatment for early-stage HCC patients however only up to $30 \%$ of them are eligible for the procedures. Natural killer (NK) cells are cytotoxic lymphocytes recognized for killing virally infected cells and improving immune functions for defending the body against malignant cells. Although autologous NK cells failed to demonstrate significant clinical benefit, transfer of allogeneic adoptive NK cells arises as a promising approach for the treatment of solid tumors. The immunosuppressive tumor microenvironment and inadequate homing efficiency of NK cells to tumors can inhibit adoptive transfer immunotherapy (ATI) efficacy. However, potential of the NK cells is challenged by the transfection efficiency. The local ablation techniques that employ thermal or chemical energy have been investigated for the destruction of solid tumors for three decades and demonstrated promising benefits for individuals not eligible for surgical resection or partial hepatectomy. Irreversible electroporation (IRE) is one of the most recent minimally invasive ablation methods that destruct the cell within the targeted region through non-thermal energy. IRE destroys the tumor cell membrane by delivering high-frequency electrical energy in short pulses and overcomes tumor immunosuppression. The previous studies demonstrated that IRE can induce immune changes which can facilitate activation of specific immune responses and improve transfection efficiency. In this review paper, we have discussed the mechanism of NK cell immunotherapy and IRE ablation methods for the treatment of HCC patients and the combinatorial benefits of NK cell immunotherapy and IRE ablation.
\end{abstract}

Keywords: Combination therapy; hepatocellular carcinoma; irreversible electroporation (IRE); natural killer cells (NK cells)

Submitted Feb 03, 2021. Accepted for publication Apr 05, 2021.

doi: $10.21037 /$ atm-21-539

View this article at: https://dx.doi.org/10.21037/atm-21-539 


\section{Introduction}

Hepatocellular carcinoma (HCC), most common type of liver cancer, makes up to $85 \%$ of liver cancer diagnoses (1). As mainly occurring due to liver cirrhosis caused by hepatitis and alcoholic cirrhosis, HCC is ranked as the fourth leading cause of cancer-related deaths worldwide (2). Despite potential prevention of the risk factors that cause HCC, HCC surveillance has limited availability and restricts the implementation of probable curative treatment options for HCC patients (2). Surgical resection and partial hepatectomy remain effective curative treatments for early-stage HCC patients, according to the BarcelonaClinic Liver Cancer (BCLC) disease classification (3). However, only up to $30 \%$ of the patients are surgical candidates due to multiple lesions related to chronic liver disease (4). Systemic chemotherapy increases the median survival time of the patients with advanced HCC by about three months $(5,6)$.

Natural killer (NK) cells are cytotoxic innate immune cells that are specialized in defense against tumors and they constitute the first line of defense against invading neoplastic cells. Early studies utilizing autologous NK cellbased adoptive transfer immunotherapy (ATI) have failed to demonstrate significant clinical benefit due to the circulation of the NK cells in the bloodstream instead of tumor structures (7). However, allogeneic adoptive transfer of NK cells collected from healthy individuals to cancer patients is assumed to be a promising approach for the treatment of solid tumors including unresectable liver tumors $(7,8)$. Yet, critical barriers, e.g., tumor immunosuppression must be overcome to achieve prominent therapeutic outcomes.

Hepatic tumor ablation, including radiofrequency ablation (RFA), microwave ablation (MWA), or cryoablation, has shown great promise for complete remission in patients with HCC smaller than $3 \mathrm{~cm}$ in diameter (9). However, the efficacy of these methods is challenged by location, size, and the number of tumors due to the "heat-sink" effect in which blood flow causes a cooling effect reducing the volume of ablated tumor region located near major blood vessels (10). Irreversible electroporation (IRE), a non-thermal tissue ablation technique, instigates cell death within the tumor structure and preserves the extracellular matrix, and induces minimal inflammation $(11,12)$. Unlike other ablation techniques, IRE induces immunogenic cell death with the use of high voltage low-energy direct current pulses delivered to the treatment region with great precision while preserving vital structures such as the extracellular matrix and blood vessels within the treatment region $(13,14)$, which is ideal for stimulating local inflammation and promoting NK cell infiltration into tumors. With the advent of immunotherapy for liver tumors, there is an increasing clinical interest in understanding the potential combination of NK cell treatment with the IRE ablation method to maximize therapeutic responses. In this review, we summarize the current investigations of NK cellbased immunotherapy and IRE ablation method as well as combination therapy (NK plus IRE) for the treatment of HCC patients.

We present the study in accordance with the Narrative Review reporting checklist (available at https://dx.doi. org/10.21037/atm-21-539).

\section{Methods}

For this study, we searched the PubMed and Web of Science databases for articles published in English during the last 20 years until August 1, 2020. The selected relevant articles were reviewed and reported in this manuscript.

\section{NK cell-based immunotherapy}

NK cells, an essential part of the innate immunity, are expressed as a member of lymphocytes that fight against tumor cells without prior sensitization (15) and are frequently located in the peritoneal cavity, bone marrow, liver, lung, lymph nodes, peripheral blood, spleen, and thymus (16). NK cells account for $20-30 \%$ of the hepatic lymphocytes in healthy human liver tissue as well as $10 \%$ of the lymphocytes in lungs (17). NK cells induce apoptosis of tumor cells through various functional mechanisms such as producing cytotoxic granules loaded with granzyme and perforin, death receptor-mediated apoptosis, secreting cytoplasmic granules, and antibodydependent cellular cytotoxicity (18). Moreover, NK cells are suitable immunotherapeutic targets for cancer treatment, e.g., adoptive cell transfer and antibody-based strategies (19). Therefore, researchers have focused on the administration of NK cells for cancer immunotherapy. The activation of NK cells is administered by inhibitory and activating receptor signals in which NK cell activation leads to the release of granules by causing cytotoxicity with the downregulation or absence of self major histocompatibility complex (MHC) class I molecules in encountered cells (Figure 1) (20,21).

Several studies have reported that the malfunction of 


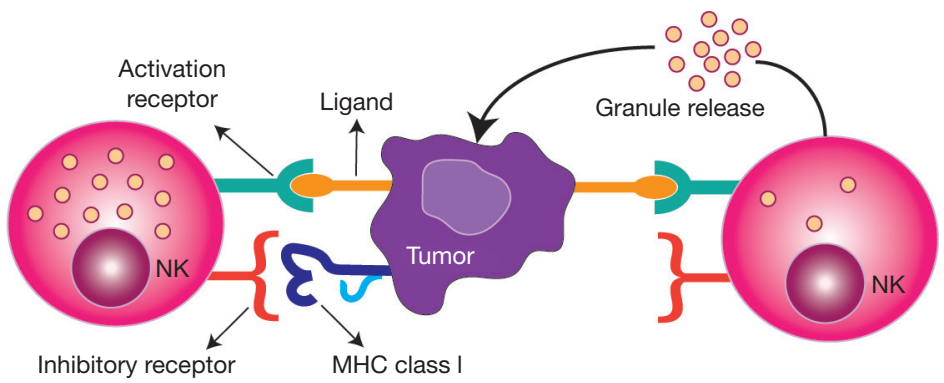

Figure 1 The activation of natural killer cells is controlled by the signals from inhibitory and activation receptors. NK cell activation is restricted as self MHC class I molecules are recognized by inhibitory NK cell receptors. In the case of downregulation or absence of self MHC class I molecules in encountered cells, NK cell activation leads to the release of granules by causing cytotoxicity. NK, natural killer; MHC, major histocompatibility complex.

NK cells was associated with the progression and metastasis of different types of cancers in both animal models and clinical studies (22-24). Particularly, increasing functional impairment and reduced proliferation of NK cells during hepatocarcinogenesis has been reported in a review article by Sun et al. (25). Meanwhile, Cai et al. observed a significant reduction of intrahepatic NK cells and a dramatic level of reduction in peripheral NK cells in HCC patients compared to healthy subjects (26). The ratio of NK cell reduction became more noticeable in the later stages of the HCC disease indicating the migration of NK cells to tumor regions $(27,28)$. In addition, frequency and cytolytic activity of NK cells have been damaged during liver cancer (25). Several approaches have been adopted to improve the efficacy of NK cell-based immunotherapy by overcoming the lack of functioning or reduced level of NK cell presence, e.g., increasing cytotoxicity of NK cells using cytokine treatment, modulating the cytotoxic function of NK cells using antibodies, boosting NK cell-activating receptors, adoptive transfer of NK cells.

The first FDA-approved cytokine, interleukin 12, improves the cytotoxicity of the NK cells while it also improves the regulatory $\mathrm{T}$ (Treg) cells, which can diminish the efficacy of the NK cells (29). The study conducted by Barajas et al. demonstrated the possibility of treating HCC with the injection of adenovirus expressing IL-12 activating NK cells and inhibiting angiogenesis (30). On the other hand, Levin et al. suggested a strategy for the modification of IL-2 to prevent activation of Treg cells while maintaining an increase of cytotoxicity of $\mathrm{NK}$ and $\mathrm{CD}^{+} \mathrm{T}$ cells (31). An in vivo study showed successful results in improving cytotoxic $\mathrm{T}$ cell expansion and therefore antitumor responses. Moreover, in vitro experiments, showed enhanced IL-2 potency, and regulated cell specificity was observed. Pillet et al. demonstrated the potential of IL-15 for improving NK cell activity and increasing the cytotoxicity of NK cells and $\mathrm{CD}^{+} \mathrm{T}$ cells without simulating Treg cells (32). Furthermore, several studies investigated the strategies utilizing cytokine gene therapies in animal models for the proliferation and activation of NK cells (33-40). Tatsumi et al. demonstrated that intrahepatic injection of alphagalactosylceramide-pulsed dendritic cells into the liver efficiently activated NK cells while inducing complete tumor rejection and increasing long-term survival benefits in a murine CMS4 tumor model (33). Leboeuf et al. have shown that human allogeneic suicide gene-modified killer cells exhibit cytotoxicity towards HCC mostly controlled by NK and NK T cells (34). Lo et al. highlighted the potential of IL-12 cytokines for the regulation of hepatic $\mathrm{T}$ cells, NK cells, and NK T cells in addition to reducing hepatic metastasis and improving survival with adenoassociated virus serotype 8/IL-12 treatment (35). The study of Gonzalez-Carmona et al. demonstrated that transduction of tumor-associated antigen-pulsed dendritic cells with a CD40L-encoding adenovirus provided significantly improved tumor infiltration with $\mathrm{CD}^{+}, \mathrm{CD} 8^{+}$ T, and NK cells (36). Abushahba et al. demonstrated that the antitumor activity of type III interferon (IFN- $\lambda$ ) was similar or better compared to type I interferon (IFN- $\alpha$ ) without a direct effect on NK cells (37). Moreover, Ebert et al. showed a strong IFN- $\alpha$ response as well as antiviral activity in liver cells introduced by $3 \mathrm{p}$ double-stranded RNA (3p-RNA) activated retinoic acid-inducible protein I (38). Guo et al. targeted Pim-3 gene by constructing a dualfunction small hairpin RNA (shRNA) vector containing an shRNA for proliferation and inhibition of apoptosis (39). 

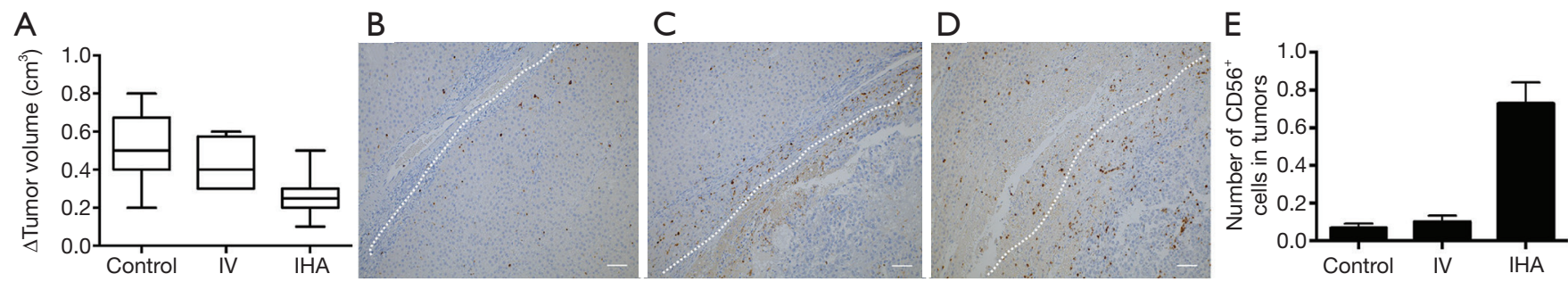

Figure 2 Natural killer (NK)-based adoptive transfer immunotherapy (ATI) outcome in a rat model of hepatocellular carcinoma (HCC). (A) The tumor volume changes after 8 days of NK cell infusion. (B,C,D) Representative CD56-stained histology slices corresponding to subjects from the control group with transcatheter intra-hepatic arterial (IHA) saline (B), intravenous (IV) NK cell (C), and transcatheter IHA NK cell infusion after 8 days of infusion (D). (E) The IHA NK cell infusion group had a significantly $(\mathrm{P}<0.0001)$ improved number of CD $56^{+}$NKs compared to the control group. (B,C,D) Scale bars $=100 \mu \mathrm{m}$. [Adapted from Su et al. (46), Copyright 2018 by John Wiley \& Sons Ltd.]

Besides, the study of Han et al. demonstrated that 5 '-end triphosphate hepatitis B virus X gene RNAs prevented replication of hepatitis $\mathrm{B}$ virus by strong expression of IFN- $\alpha$ and proinflammatory cytokines as well as activation of the retinoic acid-inducible gene I (40). These studies supported the potential benefits of using NK cells as a treatment approach and suggested further investigation before performing clinical trials.

The transfer of the autologous or allogeneic peripheral blood NK cells is another approach that has gained researchers' attention on cancer immunotherapy $(18,41)$. The efficacy of transplanted NK cells is associated with killing specificity, in vivo activity, and persistence. Several studies emphasized that autologous NK cells could not demonstrate clinical benefits in the long-term since their anti-tumor activity is limited by the inhibitory signal transmitted by self MHC molecules $(42,43)$. Parkhurst et al. used adoptively transferred in vitro activated autologous NK cells for the treatment of patients with melanoma and renal cell carcinoma following the lymphodepleting chemotherapy regimen (42). Despite no significant clinical benefit adoptively transferred NK cells were in peripheral circulation but they could not kill tumor cells in vitro without IL-2 reactivation. Sakamoto et al. treated locally advanced and/or metastatic digestive cancer patients using NK cells expanded ex-vivo with the simulation of peripheral blood mononuclear cells with OK432, IL-2, and modified FN-CH296 induced T cells (43). In addition to good-toleration of the treatment without severe adverse effects, they observed significantly increased cytotoxicity in the peripheral blood until four weeks after treatment which suggested the potential for evaluation of the approach in further clinical trials. Allogeneic NK cell infusion demonstrated an improved clinical outcome for the treatment of late-stage cancer patients including HCC $(44,45)$ in which the procedure for NK cell isolation, expansion, and purity was critical for successful results (7). Moreover, a preclinical study performed by $\mathrm{Su}$ et al. demonstrated that further improvement can be observed with a transcatheter intra-hepatic infusion of NK cells for the treatment of HCC (46). The results emphasized the significantly increased number of $\mathrm{CD} 56^{+} \mathrm{NK}$ cells in the treatment group compared to the control group in addition to reduced tumor volume growth in treated subjects (Figure 2).

The potential benefits of chemotherapy for augmenting NK cell efficacy have been investigated by several groups (5,47-49). Sorafenib, an FDA-approved multi-kinase inhibitor, is a standard treatment providing survival benefits for advanced HCC patients (5). As targeting vascular endothelial growth factor receptor and platelet-derived growth factor receptor, Sorafenib suppresses tumor cell proliferation and angiogenesis in addition to an increased rate of apoptosis in solid tumor models (5). In HCC, Sorafenib inhibits serine-threonine kinases Raf-1 and vascular endothelial growth factor pathways that administer cellular signaling for the molecular pathogenesis of HCC (50-53). Sprinzl et al. demonstrated that Sorafenib induces NK cell antitumor response through the proinflammatory activity of tumor-associated macrophages (47). In addition, Kamiya et al. demonstrated that anti-HCC cytotoxicity of NK cells was improved in HCC cell line exposed to $5 \mu \mathrm{mol} / \mathrm{L}$ of Sorafenib for 48 hours (48) which suggests potential for combined benefits of chemotherapy and immunotherapy (49). Other clinical trials have been conducted to evaluate the efficacy and safety profile of the NK cells in HCC patients and are 
schematized in Table 1. The outcome of these clinical studies will reveal better understanding of the immunotherapeutic mechanism of NK cell-based therapy for HCC and further advancement of the clinical strategies.

\section{IRE ablation}

Surgical resection and partial transplantation are preferred curative options for patients with HCC. However, they are directly affected by several factors including but not limited to tumor location and stage, patient condition, and liver functions. Therefore, a limited number of patients are suitable for these treatment approaches. The local ablation techniques, using thermal or chemical energy, have been developed in the last three decades and demonstrated benefits for the patients who are not suitable surgical candidates $(3,54)$. In addition, ablation methods became a recommended treatment approach for very early (tumor diameter $<2 \mathrm{~cm}$ ) and early-stage (tumor diameter $<3 \mathrm{~cm}$ ) HCC following the meeting of the European Association for the Study of the Liver in 2017 (3,55). RFA, first utilized in the 1990s, became the first line of ablation methods, later followed by MWA and cryoablation. Briefly, RFA utilizes thermal energy delivered to targeted lesions through radiofrequency electrodes placed under imaging guidance to destroy malignant tissues. During RFA, electric current leads to necrosis and cell death while high temperature $\left(60-100{ }^{\circ} \mathrm{C}\right)(56,57)$ limits the efficacy due to local tissue charring and heat-sink effects (56). To overcome these limitations, it was suggested to cool the electrodes internally and use multiple electrodes in the bipolar mode, despite the increased risk of bleeding and adjacent organ damages. In MWA, the heat generated by microwave energy is utilized to destroy targeted tissues similarly to RFA, but with fewer limitations (58). The design of the microwave antenna can elicit even heat transfer within the targeted region. Nevertheless, MWA has not replaced RFA despite proven benefits in comparison studies (59-61). Cryoablation, eliciting tumor necrosis, and cell death through freezing, is susceptible to the cool sink effect which limits its efficacy $(57,62)$. The limitations of these methods derived the clinical need for implementation of a nonthermal source for ablation of the tissue while eliminating heat-sink effect and allowing safe utilization of the method closer to large vessels.

IRE, a non-thermal and minimally invasive ablation method, is a relatively new ablation method that incorporates delivery of high-frequency electrical energy in short pulses to malignant tissues $(63,64)$. IRE leads to abnormal transmembrane electrical potential which increases the permeability of the cell membrane and causes irreversibly open plasma membranes leading to apoptosis (65-67). Compared to the alternatives, destruction of the cells without thermal features enables the potential application of IRE procedure without damaging close tissues, e.g., vessels, nerves, or ducts (12). Moreover, boundary between treated and untreated tissue region after the procedure can lead to a better evaluation of the treatment response by allowing easier monitoring and controlling the procedure (68). The reversible electroporation (RE) and IRE zones generated by IRE ablation can be visualized in which IRE zone includes dead cells and RE zone represents the less affected peripheral region (Figure 3). In magnetic resonance imaging (MRI), IRE zone was observed as hyperintense on $\mathrm{T} 1 \mathrm{w}$ and $\mathrm{T} 2 \mathrm{w}$ images due to the coagulative necrosis while RE zone is described as hypointense on $\mathrm{T} 1 \mathrm{w}$ and hyperintense $\mathrm{T} 2 \mathrm{w}$ MRI images (69).

The safety profile of the IRE ablation methods has been investigated by several studies (70-76). Dollinger et al. analyzed the effects of IRE on vascular structures by examining subacute ( 1 and 3 days) and midterm (average 5.7 months) follow-up data (70). In their study, vascular changes were detected in 19 vessels among 194 venous structures located in a $1 \mathrm{~cm}$ distance ablation zone in which IRE was performed on 84 hepatic lesions. The follow-up investigation demonstrated partial or complete vascular recovery on 9 of 14 cases, concluding that vascular structures located close to the IRE ablation zone were lightly affected. Another study investigated the injury of bile ducts following IRE procedure in which 55 bile ducts were examined within a $1 \mathrm{~cm}$ distance of 53 hepatic tumors (71). The follow-up MRI analyses showed that 8 of 55 bile ducts had a narrowing, 7 had dilation while no adverse events were observed on the remaining 40 bile ducts. In addition, a recent review showed that IRE is a safe alternative to perform at the tissues close to the critical structures with a lower risk of collateral damage compared to other ablation methods (37).

The efficacy of IRE was reported in studies using different animal models (12,77-80). Guo et al. performed IRE on thirty Sprague-Dawley rats with HCC and used MRI for evaluation of the therapeutic response 14 days after completion of the procedure (77). Their results suggested that IRE is an efficient method to ablate liver tumor tissue as a potential candidate for HCC treatment. Lee et al. assessed the effectiveness of 


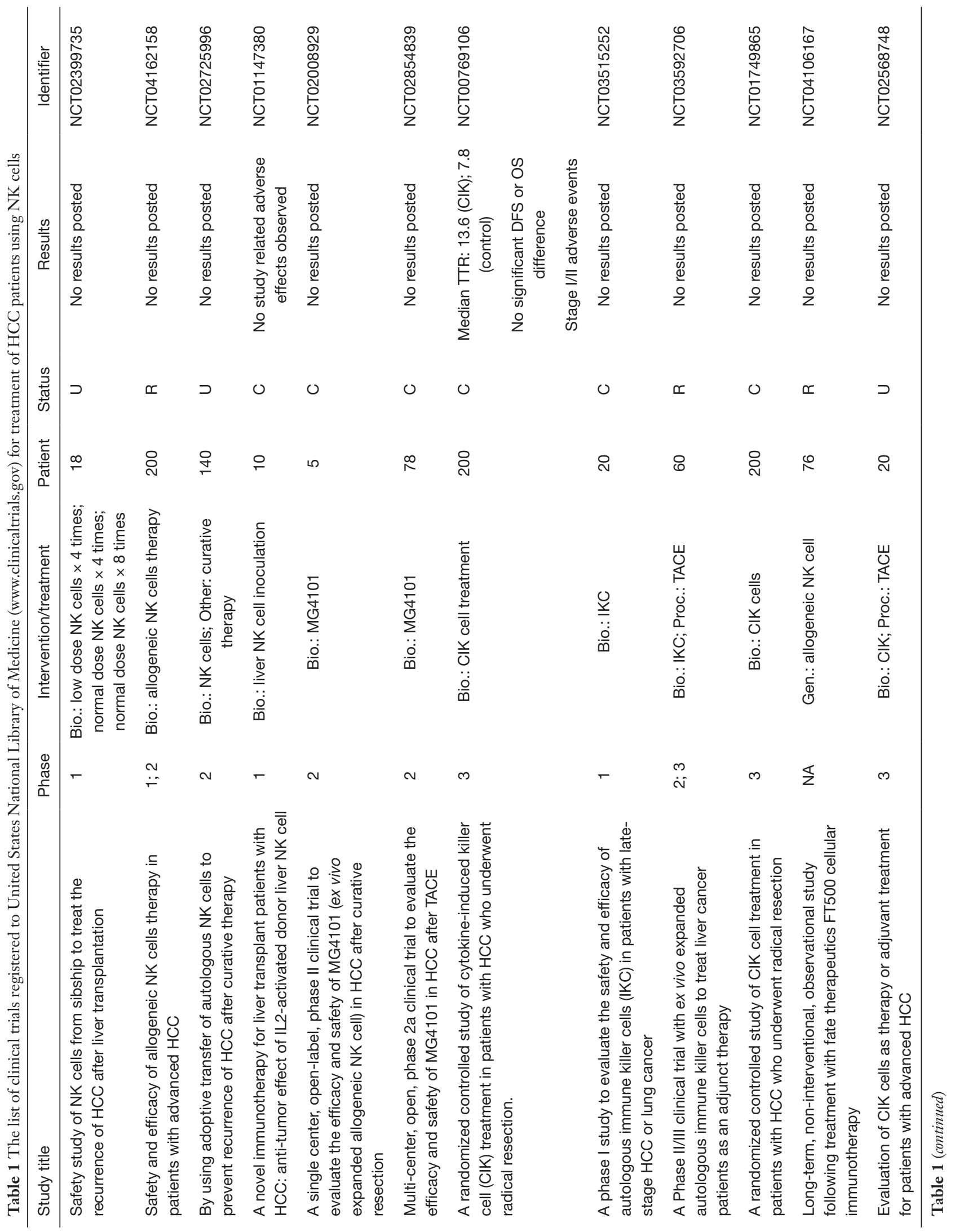




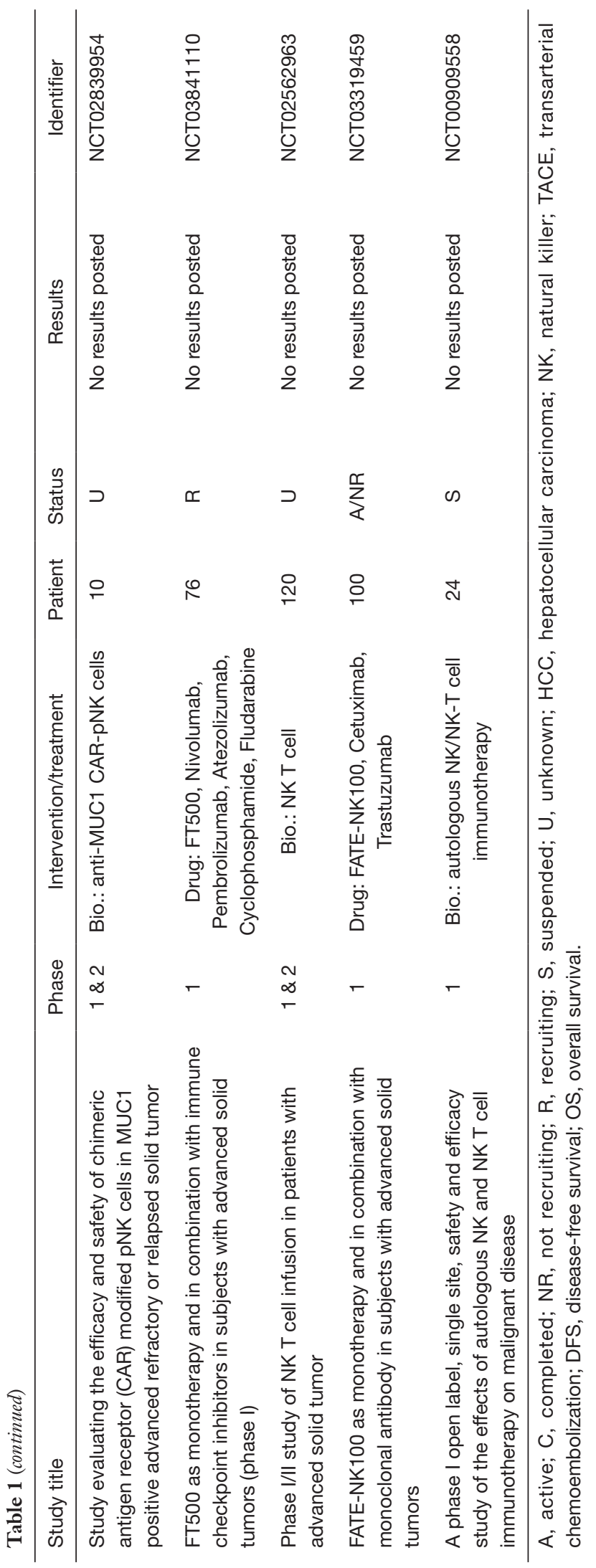




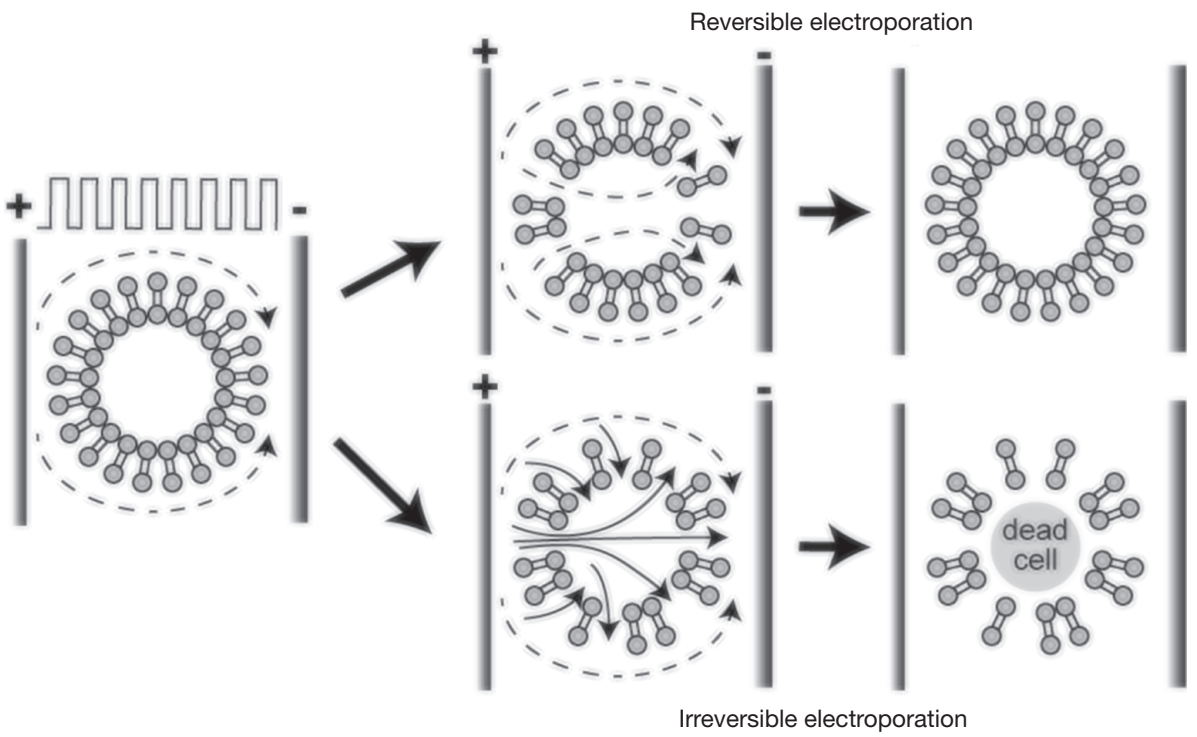

Figure 3 The representation of irreversibly and reversible electroporated zones after the irreversible electroporation (IRE) ablation procedure. Complete cell death occurs within the irreversible electroporation zone while cells in the peripheral region are minimally affected and demonstrate recovery after IRE ablation.

the IRE ablation by performing a procedure on healthy liver tissue of sixteen Yorkshire pigs (12). They did not observe any complications following the IRE procedure for 55 ablation zones of 16 subjects which suggested IRE as a safe and effective ablation approach for necrosis. In another experiment, potential clinical translation of the IRE ablation method was investigated using VX2 liver tumor model with single or multiple ablations on twenty New Zealand white rabbits (80). Several studies focused on immediate therapeutic response following IRE in animal models (78,81-87). Zhang et al. investigated the changes using MRI after IRE with different voltages in a rat liver model (78). Additional studies demonstrated that other imaging modalities (ultrasound, contrast-enhanced computed tomography (CT), diffusionweighted MRI, and contrast-enhanced MRI) have the potential for early assessment of IRE treatment effects by visualizing immediate changes following the ablation (81-85). More recently, an advanced MRI technique was used on VX2 rabbit HCC model to differentiate IRE regions from reversibly electroporated (RE) zones which will be beneficial for intraprocedural assessment $(86,87)$. The results further emphasize the potential of immediate evaluation of $\mathrm{RE}$ regions using MRI to determine the treatment strategy (Figure 4).

The clinical outcome of IRE ablation in patients with solid tumors has also been performed (73,88-98). Thomson $e t a l$. investigated the safety of the IRE technique in a single-center prospective non-randomized study including 38 patients with advanced liver, kidney, or lung malignancies and unresponsive to alternative therapies (73). The results demonstrated that IRE is a safe ablation method in clinical usage for cancer treatment. Cannon et al. performed a clinical investigation to evaluate the efficacy of IRE on hepatic tumors (88). Of 44 patients undergoing IRE treatment, five patients had 9 adverse effects which were classified as unrelated (leukocytosis, urinary tract infection), indirectly related (dehydration, biliary stent occlusion, cholangitis, and acute renal failure), procedure-related (neurogenic bladder, abdominal pain, and flank pain) and all these effects were resolved in 30 days. Moreover, the study results completed by Sugimoto et al. suggested that IRE was well tolerated by the patients with small tumor size and satisfactorily controlled disease (91). Eller et al. performed the IRE procedure on 14 patients who were not surgical candidates and had lesions near large vessels (92). Ten of the 14 patients were successfully treated without local recurrence at least for a mean of $388 \pm 160$ days. In addition, Niessen et al. discussed that patients with larger tumor volume may be poor candidates for IRE treatment due to the association of tumor volume and early disease recurrence observed in their study (93). More recent studies also demonstrated that IRE is a safe and efficient alternative ablation method for patients with unresectable tumors (96-98). 

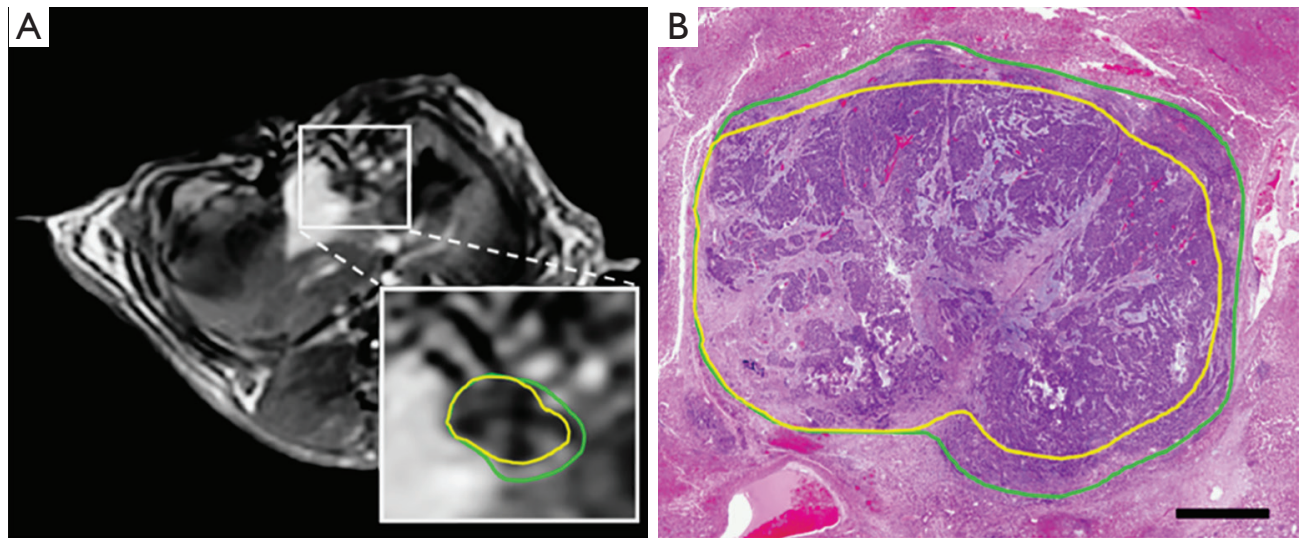

Figure 4 Magnetic resonance imaging (MRI) and histology-based irreversible electroporation (IRE) and reversible electroporation (RE) areas. (A) Representative postcontrast T1-weighted MRI where the reversibly electroporated zone is outlined in yellow and the peripheral reversibly electroporated zone in green. (B) H\&E-stained histology slide, corresponding regions were demonstrated in which the necrotic (irreversible) center is marked in yellow and the enhanced rim is emphasized in green. [Adapted from Figini et al. (87). Copyright 2018 by John Wiley \& Sons Ltd.].

Currently, there are completed and ongoing clinical trials for the assessment of efficacy and feasibility of the IRE ablation method in the treatment of HCC (Table 2). The comprehensive evaluation of the IRE procedure during these clinical trials will further present evidence for efficacy and safety-profile of IRE as well as advance our knowledge of the tumor response following ablation.

\section{IRE plus NK cell-based treatment}

Previous studies have demonstrated the clinical benefits of the IRE ablation procedure by highlighting advantages compared to thermal ablation methods including a high ablation rate and recurrence-free disease period not affected by blood flow $(10,64,88,89,99,100)$. However, several studies have demonstrated possible advancements for this local ablation method for the efficient treatment of cancer patients (101-104). Particularly, a preclinical study completed by Neal et al. suggested that immunocompetent subjects may have a better IRE therapeutic response compared to immunodeficient subjects (105). Therefore, boosting the immune system of the candidate patients remains important for improved IRE treatment outcomes. As an essential element of the innate immune system, NK cells are one of the most promising therapeutic agents to perform following IRE ablation for the treatment of solid tumors. Lin et al. investigated the safety and clinical efficacy of this potential combinative approach for the treatment of advanced-stage pancreatic cancer patients with unresectable tumors (106). Throughout this clinical trial, patients had local or systemic adverse effects measured as grade 1 (nausea andhaoba emesis, 7.04\%; puncture point pain, $29.58 \%$; fatigue, $22.54 \%$, fever, $30.99 \%$ ) or grade 2 (duodenum and gastric retention, $4.23 \%$, transient reduction of intraoperative blood pressure, $25.35 \%$; white cell count reduction, $18.31 \%$ ), and all of these side effects were resolved on the same day with symptomatic treatments without any complications. In addition, median progression-free survival and overall survival of the patients treated with a combination of IRE ablation and NK cell-based immunotherapy were significantly improved in stage III pancreatic cancer patients as well as median overall survival of stage IV patients was extended. The clinical efficacy of combination treatment for stage IV HCC was initially reported by Alnaggar et al. (45). In this study, twenty patients only received an IRE ablation while the remaining twenty patients additionally received NK cell-based immunotherapy 4-6 days after IRE ablation. The procedures were completed without severe complications and patients with mild adverse effects treated with symptomatic management. The study showed that patient cohort that received combination treatment had significantly improved median overall survival (10.1 months) compared to patients treated with a single therapy (8.9 months, $\mathrm{P}=0.0078$ ). Moreover, observation data acquired 3 months following the treatments demonstrated a significant difference in tumor size among the groups (IRE: $2.68 \pm 1.01 v s$. NK-IRE: $2.31 \pm 0.68 \mathrm{P}<0.05$ ), and combination group obtained a higher disease control rate 


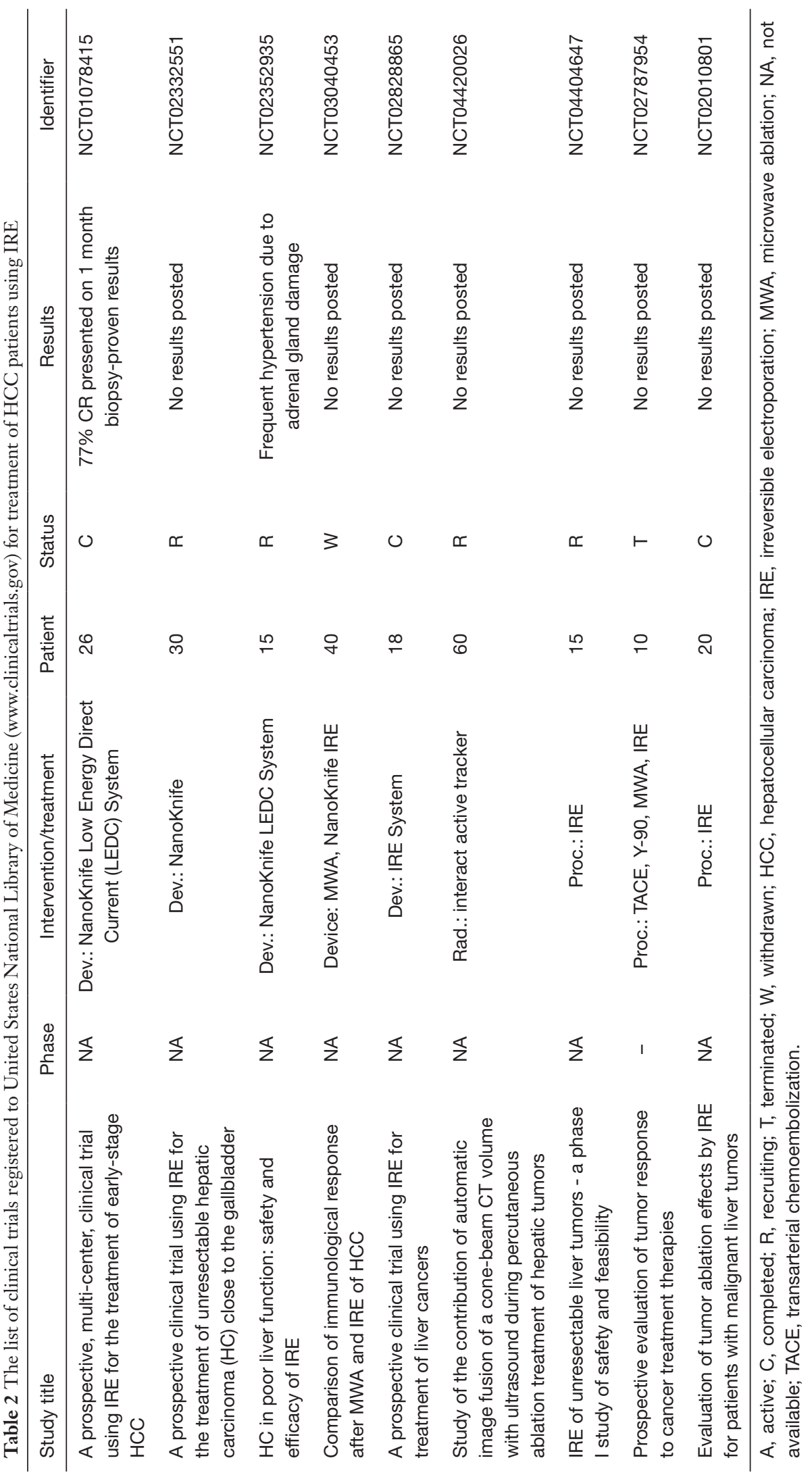


than IRE group. A recent comprehensive study reported the safety and short-term efficacy of the IRE plus NK cellbased immunotherapy for the treatment with unresectable primary liver cancer patients enrolled in the first clinical trial (NCT03008343) in collaboration with Fuda Cancer Hospital and Shenzhen Hank Bioengineering Institute (107). The results demonstrated that performing NK cellbased immunotherapy following IRE ablation generates a synergistic effect that significantly reduces the number of tumor cells in circulation and improved the immune functions of the patients. The researchers stated that $88.9 \%$ of the patients treated with the combination therapy demonstrated clinical response while $68.2 \%$ of the patients treated with only IRE ablation in three months posttreatment. Moreover, significantly improved progressionfree and overall survival was observed for the patients receiving combination treatment.

\section{Conclusions}

During the past few decades, many studies have demonstrated the potential benefits of immunotherapy for the treatment of solid tumors. As a key agent of the innate immune system, NK cells have great promise for fighting against tumor cells. Adoptive transfer of NK cells has further emphasized the potential benefits for cancer patients with solid tumors. However, immunosuppressive tumor microenvironment and inadequate homing efficiency of NK cells to tumor tissues (particularly following systemic administration) have inhibited ATI efficacy. As a minimally invasive non-thermal ablation technique, IRE instigates cell death within the applied region with minimal damage to the perpendicular region. The destruction of the tumor microenvironment by IRE enables overcoming tumor immunosuppression. Recent studies have demonstrated that a combination of IRE and NK cell-based immunotherapy has the potential to improve patient survival in the advanced-stage liver and pancreatic cancers. Besides, several studies that combine other immunotherapeutic methods with IRE ablation method have presented promising outcomes for the treatment of pancreatic tumors in which comprehensive reports will be beneficial for the researchers with the focus of cancer research (108-112). The data to be acquired from future studies will further emphasize the benefits of utilizing combination treatment of patients with HCC and also support the findings for assessment of long- term clinical response.

\section{Acknowledgments}

Funding: This study was supported by the National Cancer Institute (grants R01CA196967, R01CA209886, and R01CA241532), 2019 Harold E. Eisenberg Foundation Scholar Award, and SIR Foundation Pilot Grant (PR0000000012).

\section{Footnote}

Reporting Checklist: The authors have completed the Narrative Review reporting checklist. Available at https:// dx.doi.org/10.21037/atm-21-539

Peer Review File: Available at https://dx.doi.org/10.21037/ atm-21-539

Conflicts of Interest: All authors have completed the ICMJE uniform disclosure form (Available at https://dx.doi. org/10.21037/atm-21-539). The authors have no conflicts of interest to declare.

Ethical Statement: The authors are accountable for all aspects of the work in ensuring that questions related to the accuracy or integrity of any part of the work are appropriately investigated and resolved.

Open Access Statement: This is an Open Access article distributed in accordance with the Creative Commons Attribution-NonCommercial-NoDerivs 4.0 International License (CC BY-NC-ND 4.0), which permits the noncommercial replication and distribution of the article with the strict proviso that no changes or edits are made and the original work is properly cited (including links to both the formal publication through the relevant DOI and the license). See: https://creativecommons.org/licenses/by-nc-nd/4.0/.

\section{References}

1. Johnston MP, Khakoo SI. Immunotherapy for hepatocellular carcinoma: Current and future. World J Gastroenterol 2019;25:2977-89.

2. Yang JD, Hainaut P, Gores GJ, et al. A global view of hepatocellular carcinoma: trends, risk, prevention and management. Nat Rev Gastroenterol Hepatol 
2019;16:589-604.

3. European Association for the Study of the Liver. EASLEORTC clinical practice guidelines: management of hepatocellular carcinoma. J Hepatol 2012;56:908-43.

4. Belghiti J, Kianmanesh R. Surgical treatment of hepatocellular carcinoma. HPB (Oxford) 2005;7:42-9.

5. Llovet JM, Ricci S, Mazzaferro V, et al. Sorafenib in Advanced Hepatocellular Carcinoma. N Engl J Med 2008;359:378-90.

6. Burroughs A, Hochhauser D, Meyer T. Systemic treatment and liver transplantation for hepatocellular carcinoma: two ends of the therapeutic spectrum. Lancet Oncol 2004;5:409-18.

7. Liu P, Chen L, Zhang H. Natural Killer Cells in Liver Disease and Hepatocellular Carcinoma and the NK Cell-Based Immunotherapy. J Immunol Res 2018;2018:1206737.

8. Lim O, Jung MY, Hwang YK, et al. Present and Future of Allogeneic Natural Killer Cell Therapy. Front Immunol 2015;6:286.

9. Ryan MJ, Willatt J, Majdalany BS, et al. Ablation techniques for primary and metastatic liver tumors. World J Hepatol 2016;8:191-9.

10. Aycock KN, Davalo RV. Irreversible Electroporation: Background, Theory, and Review of Recent Developments in Clinical Oncology. Bioelectricity 2019;1:214-34.

11. Lavee J, Onik G, Mikus P, et al. A novel nonthermal energy source for surgical epicardial atrial ablation: irreversible electroporation. Heart Surg Forum 2007;10:E162-7.

12. Lee EW, Chen C, Prieto VE, et al. Advanced hepatic ablation technique for creating complete cell death: irreversible electroporation. Radiology 2010;25 5:426-33.

13. Zimmerman A, Grand D, Charpentier KP. Irreversible electroporation of hepatocellular carcinoma: patient selection and perspectives. J Hepatocell Carcinoma 2017;4:49-58.

14. Charpentier KP. Irreversible electroporation for the ablation of liver tumors: are we there yet? Arch Surg 2012;147:1053-61.

15. Peng H, Tian Z. Natural Killer Cell Memory: Progress and Implications. Front Immunol 2017;8:1143.

16. Mandal A, Viswanathan C. Natural killer cells: In health and disease. Hematol Oncol Stem Cell Ther 2015;8:47-55.

17. Bonanni V, Sciumè G, Santoni A, et al. Bone Marrow NK Cells: Origin, Distinctive Features, and Requirements for Tissue Localization. Front Immunol 2019;10:1569.

18. Cheng M, Chen Y, Xiao W, et al. NK cell-based immunotherapy for malignant diseases. Cell Mol Immunol 2013;10:230-52.

19. Levy EM, Roberti MP, Mordoh J. Natural Killer Cells in Human Cancer: From Biological Functions to Clinical Applications. J Biomed Biotechnol 2011;2011:676198.

20. Paul S, Lal G. The Molecular Mechanism of Natural Killer Cells Function and Its Importance in Cancer Immunotherapy. Front Immunol 2017;8:1124.

21. Freund J, May RM, Yang E, et al. Activating Receptor Signals Drive Receptor Diversity in Developing Natural Killer Cells. PLoS Biol 2016;14:e1002526.

22. Guerra N, Tan YX, Joncker NT, et al. NKG2D-deficient mice are defective in tumor surveillance in models of spontaneous malignancy. Immunity 2008;28:571-80. Erratum in: Immunity. 2008 May;28(5):723. Greenberg, Norman R [corrected to Greenberg, Norman M].

23. Cerwenka A, Lanier LL. Natural killer cells, viruses and cancer. Nat Rev Immunol 2001;1:41-9.

24. Jun E, Song AY, Choi JW, et al. Progressive Impairment of NK Cell Cytotoxic Degranulation Is Associated With TGF- $\beta 1$ Deregulation and Disease Progression in Pancreatic Cancer. Front Immunol 2019;10:1354.

25. Sun C, Sun HY, Xiao WH, et al. Natural killer cell dysfunction in hepatocellular carcinoma and NK cell-based immunotherapy. Acta Pharmacol Sin 2015;36:1191-9.

26. Cai L, Zhang Z, Zhou L, et al. Functional impairment in circulating and intrahepatic NK cells and relative mechanism in hepatocellular carcinoma patients. Clin Immunol 2008;129:428-37.

27. Guo CL, Yang HC, Yang XH, et al. Associations between infiltrating lymphocyte subsets and hepatocellular carcinoma. Asian Pac J Cancer Prev 2012;13:5909-13.

28. Wu Y, Kuang DM, Pan WD, et al. Monocyte/ macrophage-elicited natural killer cell dysfunction in hepatocellular carcinoma is mediated by CD48/2B4 interactions. Hepatology 2013;57:1107-16.

29. Trinchieri G. Interleukin-12 and the regulation of innate resistance and adaptive immunity. Nat Rev Immunol 2003;3:133-46.

30. Barajas M, Mazzolini G, Genové G, et al. Gene therapy of orthotopic hepatocellular carcinoma in rats using adenovirus coding for interleukin 12 . Hepatology 2001;33:52-61.

31. Levin AM, Bates DL, Ring AM, et al. Exploiting a natural conformational switch to engineer an interleukin-2 'superkine'. Nature 2012;484:529-33.

32. Pillet AH, Thèze J, Rose T. Interleukin (IL)-2 and IL-15 
have different effects on human natural killer lymphocytes. Hum Immunol 2011;72:1013-7.

33. Tatsumi T, Takehara T, Yamaguchi S, et al. Intrahepatic delivery of alpha-galactosylceramide-pulsed dendritic cells suppresses liver tumor. Hepatology 2007;45:22-30.

34. Leboeuf C, Mailly L, Wu T, et al. In vivo proof of concept of adoptive immunotherapy for hepatocellular carcinoma using allogeneic suicide gene-modified killer cells. Mol Ther 2014;22:634-44.

35. Lo CH, Chang CM, Tang SW, et al. Differential antitumor effect of interleukin-12 family cytokines on orthotopic hepatocellular carcinoma. J Gene Med 2010;12:423-34.

36. Gonzalez-Carmona MA, Lukacs-Kornek V, Timmerman A, et al. CD40ligand-expressing dendritic cells induce regression of hepatocellular carcinoma by activating innate and acquired immunity in vivo. Hepatology 2008;48:157-68.

37. Abushahba W, Balan M, Castaneda I, et al. Antitumor activity of type I and type III interferons in BNL hepatoma model. Cancer Immunol Immunother 2010;59:1059-71.

38. Ebert G, Poeck H, Lucifora J, et al. 5' Triphosphorylated Small Interfering RNAs Control Replication of Hepatitis $\mathrm{B}$ Virus and Induce an Interferon Response in Human Liver Cells and Mice. Gastroenterology 2011;141:696706, 706.e1-3.

39. Guo Q, Lan P, Yu X, et al. Immunotherapy for hepatoma using a dual-function vector with both immunostimulatory and pim-3-silencing effects. Mol Cancer Ther 2014;13:1503-13.

40. Han Q, Zhang C, Zhang J, et al. Reversal of hepatitis B virus-induced immune tolerance by an immunostimulatory $3 \mathrm{p}-\mathrm{HBx}$-siRNAs in a retinoic acid inducible gene I-dependent manner. Hepatology 2011;54:1179-89.

41. Cheng M, Zhang J, Jiang W, et al. Natural killer cell lines in tumor immunotherapy. Front Med 2012;6:56-66.

42. Parkhurst MR, Riley JP, Dudley ME, et al. Adoptive transfer of autologous natural killer cells leads to high levels of circulating natural killer cells but does not mediate tumor regression. Clin Cancer Res 2011;17:6287-97.

43. Sakamoto N, Ishikawa T, Kokura S, et al. Phase I clinical trial of autologous NK cell therapy using novel expansion method in patients with advanced digestive cancer. J Transl Med 2015;13:277.

44. Lin M, Liang S, Wang X, et al. Cryoablation combined with allogenic natural killer cell immunotherapy improves the curative effect in patients with advanced hepatocellular cancer. Oncotarget 2017;8:81967-77.
45. Alnaggar M, Lin M, Mesmar A, et al. Allogenic Natural Killer Cell Immunotherapy Combined with Irreversible Electroporation for Stage IV Hepatocellular Carcinoma: Survival Outcome. Cell Physiol Biochem 2018;48:1882-93.

46. Su Z, Wang X, Zheng L, et al. MRI-guided interventional natural killer cell delivery for liver tumor treatment. Cancer Med 2018;7:1860-9.

47. Sprinzl MF, Reisinger F, Puschnik A, et al. Sorafenib perpetuates cellular anticancer effector functions by modulating the crosstalk between macrophages and natural killer cells. Hepatology 2013;57:2358-68.

48. Kamiya T, Chang YH, Campana D. Expanded and Activated Natural Killer Cells for Immunotherapy of Hepatocellular Carcinoma. Cancer Immunol Res 2016;4:574-81.

49. Hosseinzadeh F, Verdi J, Ai J, et al. Combinational immune-cell therapy of natural killer cells and sorafenib for advanced hepatocellular carcinoma: a review. Cancer Cell Int 2018;18:133.

50. Ito Y, Sasaki Y, Horimoto M, et al. Activation of mitogenactivated protein kinases/extracellular signal-regulated kinases in human hepatocellular carcinoma. Hepatology 1998;27:951-8.

51. Villanueva A, Newell P, Chiang DY, et al. Genomics and signaling pathways in hepatocellular carcinoma. Semin Liver Dis 2007;27:55-76.

52. Calvisi DF, Ladu S, Gorden A, et al. Ubiquitous Activation of Ras and Jak/Stat Pathways in Human HCC. Gastroenterology 2006;130:1117-28.

53. Semela D, Dufour JF. Angiogenesis and hepatocellular carcinoma. J Hepatol 2004;41:864-80.

54. Grandhi MS, Kim AK, Ronnekleiv-Kelly SM, et al. Hepatocellular carcinoma: From diagnosis to treatment. Surg Oncol 2016;25:74-85.

55. Forner A, Reig M, Bruix J. Hepatocellular carcinoma. Lancet 2018;391:1301-14.

56. Hinshaw JL, Lubner MG, Ziemlewicz TJ, et al. Percutaneous Tumor Ablation Tools: Microwave, Radiofrequency, or Cryoablation-What Should You Use and Why? RadioGraphics 2014;34:1344-62.

57. Krokidis ME, Kitrou P, Spiliopoulos S, et al. Imageguided minimally invasive treatment for small renal cell carcinoma. Insights Imaging 2018;9:385-90.

58. Dou JP, Liang P, Yu J. Microwave ablation for liver tumors. Abdom Radiol (NY) 2016;41:650-8.

59. Facciorusso A, Di Maso M, Muscatiello N. Microwave ablation versus radiofrequency ablation for the treatment of hepatocellular carcinoma: A systematic review and 
meta-analysis. Int J Hyperthermia 2016;32:339-44.

60. Lahat E, Eshkenazy R, Zendel A, et al. Complications after percutaneous ablation of liver tumors: a systematic review. Hepatobiliary Surg Nutr 2014;3:317-23.

61. Ding J, Jing X, Liu J, et al. Complications of thermal ablation of hepatic tumours: Comparison of radiofrequency and microwave ablative techniques. Clin Radiol 2013;68:608-15.

62. Dunne RM, Shyn PB, Sung JC, et al. Percutaneous treatment of hepatocellular carcinoma in patients with cirrhosis: A comparison of the safety of cryoablation and radiofrequency ablation. Eur J Radiol 2014;83:632-8.

63. Rubinsky B, Onik G, Mikus P. Irreversible electroporation: a new ablation modality--clinical implications. Technol Cancer Res Treat 2007;6:37-48.

64. Al-Sakere B, André F, Bernat C, et al. Tumor ablation with irreversible electroporation. PloS One 2007;2:e1135.

65. Weiss MJ, Wolfgang CL. Irreversible electroporation: a novel therapy for stage III pancreatic cancer. Adv Surg 2014;48:253-8.

66. Davalos RV, Mir LM, Rubinsky B. Tissue Ablation with Irreversible Electroporation. Ann Biomed Eng 2005;33:223.

67. Kourounis G, Paul Tabet P, Moris D, et al. Irreversible electroporation (Nanoknife ${ }^{\circledR}$ treatment) in the field of hepatobiliary surgery: Current status and future perspectives. J BUON 2017;22:141-9.

68. Lencioni R. Loco-regional treatment of hepatocellular carcinoma. Hepatology 2010;52:762-73.

69. Felker ER, Dregely I, Chung DJ, et al. Irreversible Electroporation: Defining the MRI Appearance of the Ablation Zone With Histopathologic Correlation in a Porcine Liver Model. AJR Am J Roentgenol 2017;208:1141-6.

70. Dollinger M, Müller-Wille R, Zeman F, et al. Irreversible Electroporation of Malignant Hepatic Tumors-Alterations in Venous Structures at Subacute FollowUp and Evolution at Mid-Term Follow-Up. PLoS One 2015;10:e135773.

71. Dollinger M, Zeman F, Niessen C, et al. Bile Duct Injury after Irreversible Electroporation of Hepatic Malignancies: Evaluation of MR Imaging Findings and Laboratory Values. J Vasc Interv Radiol 2016;27:96-103.

72. Dollinger M, Beyer LP, Haimerl M, et al. Adverse effects of irreversible electroporation of malignant liver tumors under CT fluoroscopic guidance: a single-center experience. Diagn Interv Radiol 2015;21:471-5.

73. Thomson KR, Cheung W, Ellis SJ, et al. Investigation of the safety of irreversible electroporation in humans. J Vasc Interv Radiol 2011;22:611-21.

74. Kingham TP, Karkar AM, D'Angelica MI, et al. Ablation of perivascular hepatic malignant tumors with irreversible electroporation. J Am Coll Surg 2012;215:379-87.

75. Silk MT, Wimmer T, Lee KS, et al. Percutaneous ablation of peribiliary tumors with irreversible electroporation. J Vasc Interv Radiol 2014;25:112-8.

76. Narayanan G, Bhatia S, Echenique A, et al. Vessel patency post irreversible electroporation. Cardiovasc Intervent Radiol 2014;37:1523-9.

77. Guo Y, Zhang Y, Klein R, et al. Irreversible electroporation therapy in the liver: longitudinal efficacy studies in a rat model of hepatocellular carcinoma. Cancer Res 2010;70:1555-63.

78. Zhang Y, Guo Y, Ragin AB, et al. MR imaging to assess immediate response to irreversible electroporation for targeted ablation of liver tissues: preclinical feasibility studies in a rodent model. Radiology 2010;256:424-32.

79. Charpentier KP, Wolf F, Noble L, et al. Irreversible electroporation of the liver and liver hilum in swine. HPB (Oxford) 2011;13:168-73.

80. Lee EW, Wong D, Tafti BA, et al. Irreversible electroporation in eradication of rabbit VX2 liver tumor. J Vasc Interv Radiol 2012;23:833-40.

81. Zhang Y, White SB, Nicolai JR, et al. Multimodality imaging to assess immediate response to irreversible electroporation in a rat liver tumor model. Radiology 2014;271:721-9.

82. Appelbaum L, Ben-David E, Sosna J, et al. US Findings after Irreversible Electroporation Ablation: RadiologicPathologic Correlation. Radiology 2012;262:117-25.

83. Wang X, Su Z, Lyu T, et al. (18)F-FDG PET Biomarkers Help Detect Early Metabolic Response to Irreversible Electroporation and Predict Therapeutic Outcomes in a Rat Liver Tumor Model. Radiology 2018;287:137-45.

84. Hu S, Sun C, Wang B, et al. Diffusion-Weighted MR Imaging to Evaluate Immediate Response to Irreversible Electroporation in a Rabbit VX2 Liver Tumor Model. J Vasc Interv Radiol 2019;30:1863-9.

85. Shangguan AJ, Sun C, Wang B, et al. DWI and DCE-MRI approaches for differentiating reversibly electroporated penumbra from irreversibly electroporated ablation zones in a rabbit liver model. Am J Cancer Res 2019;9:1982-94.

86. Pan L, Sun C, Zhou K, et al. Transcatheter Intraarterial Perfusion MRI Approaches to Differentiate Reversibly Electroporated Penumbra From Irreversibly Electroporated Zones in Rabbit Liver. Acad Radiol 
2020;27:1727-33.

87. Figini M, Zhou K, Pan L, et al. Transcatheter intra-arterial perfusion (TRIP)-MRI biomarkers help detect immediate response to irreversible electroporation of rabbit VX2 liver tumor. Magn Reson Med 2020;84:365-74.

88. Cannon R, Ellis S, Hayes D, et al. Safety and early efficacy of irreversible electroporation for hepatic tumors in proximity to vital structures. J Surg Oncol 2013;107:544-9.

89. Cheng RG, Bhattacharya R, Yeh MM, et al. Irreversible Electroporation Can Effectively Ablate Hepatocellular Carcinoma to Complete Pathologic Necrosis. J Vasc Interv Radiol 2015;26:1184-8.

90. Gonzalez-Beicos A, Venkat S, Songrug T, et al. Irreversible Electroporation of Hepatic and Pancreatic Malignancies: Radiologic-Pathologic Correlation. Tech Vasc Interv Radiol 2015;18:176-82.

91. Sugimoto K, Moriyasu F, Kobayashi Y, et al. Irreversible electroporation for nonthermal tumor ablation in patients with hepatocellular carcinoma: initial clinical experience in Japan. Jpn J Radiol 2015;33:424-32.

92. Eller A, Schmid A, Schmidt J, et al. Local Control of Perivascular Malignant Liver Lesions Using Percutaneous Irreversible Electroporation: Initial Experiences. Cardiovasc Intervent Radiol 2015;38:152-9.

93. Niessen C, Igl J, Pregler B, et al. Factors Associated with Short-Term Local Recurrence of Liver Cancer after Percutaneous Ablation Using Irreversible Electroporation: A Prospective Single-Center Study. J Vasc Interv Radiol 2015;26:694-702.

94. Granata V, de Lutio di Castelguidone E, Fusco R, et al. Irreversible electroporation of hepatocellular carcinoma: preliminary report on the diagnostic accuracy of magnetic resonance, computer tomography, and contrast-enhanced ultrasound in evaluation of the ablated area. Radiol Med 2016;121:122-31.

95. Tian G, Zhao Q, Chen F, et al. Ablation of hepatic malignant tumors with irreversible electroporation: A systematic review and meta-analysis of outcomes. Oncotarget 2017;8:5853-60.

96. Kalra N, Gupta P, Gorsi U, et al. Irreversible Electroporation for Unresectable Hepatocellular Carcinoma: Initial Experience. Cardiovasc Intervent Radiol 2019;42:584-90.

97. Wang W, Hou S, Ni J, et al. Effectiveness and safety of irreversible electroporation for recurrent hepatocellular carcinoma ineligible for thermal ablation after surgery. Journal of Interventional Medicine 2020;3:151-5.

98. Mafeld S, Wong JJ, Kibriya N, et al. Percutaneous
Irreversible Electroporation (IRE) of Hepatic Malignancy: A Bi-institutional Analysis of Safety and Outcomes. Cardiovasc Intervent Radiol 2019;42:577-83.

99. Bulvik BE, Rozenblum N, Gourevich S, et al. Irreversible Electroporation versus Radiofrequency Ablation: A Comparison of Local and Systemic Effects in a SmallAnimal Model. Radiology 2016;280:413-24.

100. Chen X, Ren Z, Zhu T, et al. Electric Ablation with Irreversible Electroporation (IRE) in Vital Hepatic Structures and Follow-up Investigation. Sci Rep 2015;5:16233.

101.He C, Huang X, Zhang Y, et al. Comparison of Survival Between Irreversible Electroporation Followed by Chemotherapy and Chemotherapy Alone for Locally Advanced Pancreatic Cancer. Front Oncol 2020;10:6.

102. Ma YY, Shi JJ, Chen JB, et al. Irreversible electroporation for liver metastasis from pancreatic cancer: A case report. World J Clin Cases 2020;8:390-7.

103. He C, Li S. Irreversible electroporation followed by chemotherapy vs. chemotherapy alone for locally advanced pancreatic cancer: A large cohort propensity score analysis. J Glob Oncol 2019;5: abstr 117.

104. Scheffer HJ, Stam AGM, Geboers B, et al. Irreversible electroporation of locally advanced pancreatic cancer transiently alleviates immune suppression and creates a window for antitumor $\mathrm{T}$ cell activation. Oncoimmunology 2019;8:1652532.

105. Neal RE 2nd, Rossmeisl JH Jr, Robertson JL, et al. Improved local and systemic anti-tumor efficacy for irreversible electroporation in immunocompetent versus immunodeficient mice. PLoS One 2013;8:e64559.

106.Lin M, Alnaggar M, Liang S, et al. An important discovery on combination of irreversible electroporation and allogeneic natural killer cell immunotherapy for unresectable pancreatic cancer. Oncotarget 2017;8:101795-807.

107. Yang Y, Qin Z, Du D, et al. Safety and Short-Term Efficacy of Irreversible Electroporation and Allogenic Natural Killer Cell Immunotherapy Combination in the Treatment of Patients with Unresectable Primary Liver Cancer. Cardiovasc Intervent Radiol 2019;42:48-59.

108. Lin M, Zhang X, Liang S, et al. Irreversible electroporation plus allogenic $\mathrm{V} \gamma 9 \mathrm{~V} \delta 2 \mathrm{~T}$ cells enhances antitumor effect for locally advanced pancreatic cancer patients. Signal Transduct Target Ther 2020;5:215.

109.Zhao J, Wen X, Tian L, et al. Irreversible electroporation reverses resistance to immune checkpoint blockade in pancreatic cancer. Nat Commun 2019;10:899. 


\section{Page 16 of 16}

110. Go EJ, Yang H, Chon HJ, et al. Combination of Irreversible Electroporation and STING Agonist for Effective Cancer Immunotherapy. Cancers (Basel) 2020;12:3123.

111. Slaughter JR, Burbach BJ, O'Flanagan SD, et al. Tumor Ablation by Irreversible Electroporation (IRE) Augments CTLA-4 Checkpoint Inhibitor Immunotherapy. J Am Coll

\section{Eresen et al. NK plus IRE ablation for treatment of patients with HCC}

Surg 2019;229:e204.

112. Narayanan JSS, Ray P, Hayashi T, et al. Irreversible Electroporation Combined with Checkpoint Blockade and TLR7 Stimulation Induces Antitumor Immunity in a Murine Pancreatic Cancer Model. Cancer Immunol Res 2019;7:1714.

Cite this article as: Eresen A, Yang J, Scotti A, Cai K, Yaghmai V, Zhang Z. Combination of natural killer cellbased immunotherapy and irreversible electroporation for the treatment of hepatocellular carcinoma. Ann Transl Med 2021;9(13):1089. doi: 10.21037/atm-21-539 